\title{
SnSe Solar Cells: Current Results and Perspectives
}

\author{
Paul Beltrán-Bobadilla, ${ }^{a}$ A. Carrillo-Osuna, ${ }^{b}$ J. A. Rodriguez-Valverde, ${ }^{b}$ B. Acevedo-Juárez, ${ }^{a}$ \\ I. Montoya De Los Santos, ${ }^{c}$ F. J. Sánchez-Rodriguez, ${ }^{b}$ and Maykel Courel ${ }^{a}$ \\ ${ }^{a}$ Centro Universitario de los Valles (CUValles), Universidad de Guadalajara. Carretera Guadalajara-Ameca \\ Km. 45.5, C.P. 46600, Ameca, Jalisco, México \\ ${ }^{b}$ Facultad de Ciencias Físico-Matemáticas, Universidad Autónoma de Sinaloa, C.P. 80010, Culiacán, \\ Sinaloa, México \\ ${ }^{c}$ Instituto de Estudios de la Energía, Universidad del Istmo, C.P. 70760, Santo Domingo Tehuantepec, \\ Oaxaca, México
}

Email: maykel.courel@academicos.udg.mx (M. C.)

\begin{abstract}
This work presents current advances and perspectives on SnSe thin film solar cell technology. Nowadays, SnSe solar cells have not been able to achieve efficiency values higher than $7 \%$. In this sense, it is necessary to study the potentiality of SnSe compound in solar cells that could help to understand further routes to promote this technology. It is demonstrated that efficiencies about $25 \%$ are expected under the ideal conditions of a low density of defects at SnSe bulk, the SnSe/buffer interface and the use of a buffer layer with a high band-gap, so that most photons get absorbed in the SnSe material with a good lattice matching to the SnSe and the negligible contribution of resistances. The comparison of our results with the one experimentally reported demonstrates that $J_{\mathrm{sc}}$ values constitute the first main issue to be solved in this technology.
\end{abstract}

Keywords SnSe solar cells, theoretical calculations, radiative limit, limiting factors

The current thin film solar cell technology is dominated by absorber compounds such as CdTe and CulnGaSe 2 . However, the toxicity of $\mathrm{Cd}$ together with the low abundance of In, $\mathrm{Ga}$, and $\mathrm{Te}$ in the Earth's crust has raised the need to study new compounds to replace them. Recently, compounds such as kesterites and perovskites have been widely studied and proposed for replacing previous technologies in solar cell fabrication due to their adequate physical properties for solar cell processing. However, the formation of defects, secondary phases, and poor band-alignment have been identified as main drawbacks concerning the Kesterites technology, resulting in efficiency values lower than $12.6 \%{ }^{[1]}$ On the other hand, despite high efficiencies that have been achieved in perovskite solar cells, the toxicity of $\mathrm{Pb}$ and the instability of the compound remain as the main concerns to be further studied. ${ }^{[2]}$ Therefore, other materials based on abundant and low toxic elements, fulfilling the basic properties for solar cell applications are being studied.

The SnSe semiconductor is among the new compounds that have been currently studied for solar cell applications. This compound consists of elements with a relative abundance in the Earth's crust and low toxicity. It also shows p-type conductivity, direct band-gap transitions with an absorption coefficient higher than $10^{4} \mathrm{~cm}^{-1}$, and a band-gap near $1.0 \mathrm{eV}{ }^{[3]}$ It also presents an orthorhombic structure (spatial group Pnma), with lattice constant values of $a=11.52 \AA, b=4.16 \AA$, and $c=$ $4.42 \AA .{ }^{[4]}$ The $p$-type conductivity of SnSe is mainly a result of Sn vacancies $\left(V_{S n}\right)$, therefore, there is no need for doping this compound with extra elements. ${ }^{[5]}$ Another important feature is that unlike kesterite compounds, better control of the phase is expected as a result of the two constituent elements, while in the case of kesterite materials consisting of at least four elements, secondary phases are prone to be formed. ${ }^{[1]}$

The SnSe compound has been deposited by many physical and chemical deposition techniques. The main physical deposition methods for SnSe material are the two-stage process and evaporation, whereas chemical bath deposition, electrodeposition, and spray pyrolysis stand for chemical routes. $^{[6]}$ Each technique has its own advantages and disadvantages. Physical techniques such as the described above relatively depend on few parameters and allow to finely control crystallinity as well as morphological and electrical properties. On the other hand, chemical routes depend on a greater number of parameters, and the effect they have on film growth and properties is less predictable than in physical routes. However, in general, low-cost deposition techniques are used for SnSe deposition, which can result in reduced values of cost per watt peak.

The first report on the fabrication of SnSe solar cells was presented in 1990 by Singh et al., ${ }^{[7]}$ where authors deposited $\mathrm{SnO}_{2}: \mathrm{F}$ (FTO) onto a glass substrate by spray pyrolysis and later cleaned it chemically and ultrasonically, followed by the sequential deposition of Se and SnSe by thermal evaporation and finally a layer of $\mathrm{Ag}$ was deposited on SnSe, as the contact. The maximum efficiency reported was $2.3 \%$ with an opencircuit voltage, short-circuit current density and fill factor (FF) values of $0.41 \mathrm{~V}, 9.2 \mathrm{~mA} / \mathrm{cm}^{2}$ and 0.49 , respectively. $I-V$ characteristics were mainly attributed to the variation of the Se layer thickness. FTO/Se/SnSe heterojunctions with thinner Se layers showed a better efficiency, greater fill factor as well as a greater open-circuit voltage. However, poor values of shortcircuit current density were obtained for these thicknesses, so non-specified improvement in fabrication parameters were suggested to increase efficiency. The results on open-circuit voltage $\left(V_{o c}\right)$, short-circuit current density $\left(J_{s c}\right)$, fill factor $(F F)$, and efficiency $(\eta)$ of processed cells are summarized in Table 1.

Mathews fabricated the first inorganic solar cell with CdS as the $n$-type buffer layer, following the $\mathrm{FTO} / \mathrm{CdS} / \mathrm{SnSe} / g r a p h i t e$ configuration, reporting a $J_{\mathrm{sc}}$ of $0.7 \mathrm{~mA} / \mathrm{cm}^{2}$, a $V_{\mathrm{oc}}$ of $140 \mathrm{mV}$, 


\section{Perspective}

Table 1 The results on $V_{\mathrm{oc}}, \mathrm{J}_{\mathrm{sc}}, \mathrm{FF}$, and $\eta$ of SnSe solar cells processed under the glass substrate/FTO/Se/SnSe/Ag configuration $^{[7]}$

\begin{tabular}{ccccc}
\hline Number of cells & $V_{\text {oc }} / \mathrm{V}$ & $J_{\mathrm{sd}}\left(\mathrm{mA} / \mathrm{cm}^{2}\right)$ & $\mathrm{FF}$ & $\eta / \%$ \\
\hline FTTS-3 & 0.41 & 9.2 & 0.49 & 2.3 \\
FTTS-9 & 0.39 & 10.8 & 0.43 & 2.2 \\
FTTS-12 & 0.33 & 12.4 & 0.37 & 1.9 \\
\hline
\end{tabular}

and conversion efficiency of $0.03 \%$ under the illumination of $100 \mathrm{~mW} / \mathrm{cm}^{2}{ }^{[8]}$ The author quoted that such a reduced efficiency value is likely to be related to a high density of defects, resulting in a high recombination rate at the $\mathrm{CdS} / \mathrm{SnSe}$ interface.

Other works have been published on the application of $\mathrm{SnSe}$ thin film in solar cells. Shinde et al. fabricated solar cells with different configurations. For ITO/CdS (100 nm)/SnSe $(800$ $\mathrm{nm}$ //polysulfide/Pt/FTO configuration, where SnSe was grown by electrodeposition, an efficiency of $1.4 \%$ was reported. ${ }^{[9]}$ This value was higher than the one obtained for a solar cell with ITO/CdS $(100 \mathrm{~nm}) / \mathrm{SnSe}(800 \mathrm{~nm}) / \mathrm{Au}(100 \mathrm{~nm})$ configuration of $0.8 \%$ under $100 \mathrm{~mW} / \mathrm{cm}^{2}$. According to Shinde et al., the photovoltaic performance is influenced by the conductivity, compactness, and uniformity of the film as well as the quality of the contact between layers. Polysulfide electrolytes presenting in the liquid junction significantly increased the efficiency since the conductivity was improved and consequently, the recombination losses were reduced. Better performance with respect to previous works was achieved by improving the quality of the junction to prevent leakage current and recombination at the grain boundaries, which increased fill factor (FF) and open-circuit voltage $\left(V_{o c}\right)$ values. However, it was suggested to optimize parameters such as layer thickness, annealing temperature, and device structure to improve the characteristics of SnSe-based devices. On the other hand, Makori et al. reported the fabrication of solar cells with glass substrate/Ag/CdO:Sn/SnSe (148 nm)/Ag structure, where SnSe was deposited by thermal evaporation, resulting in a short-circuit current, open-circuit voltage, fill factor and efficiency values of $0.993 \mathrm{~mA}, 273 \mathrm{mV}, 0.69$ and $0.59 \%$, respectively. ${ }^{[10]}$ Although the physical phenomena behind such characteristics were not described, the authors suggested three possible routes to increase the conversion efficiency in $\mathrm{SnSe}$ solar cells including optimizing the deposition temperature of the SnSe films, designing multi-layered solar cells using SnSe, and varying the ohmic contact materials.

So far, one of the highest SnSe solar cell efficiencies reported in the literature was presented by Abd El-Rahman et al., where $\mathrm{Si}$ is used as the $n$-type semiconductor buffer layer. ${ }^{[11]}$ Under an illumination source of $50 \mathrm{~mW} / \mathrm{cm}^{2}$, values of efficiency, open-circuit voltage, short-circuit current density, and fill factor of $6.44 \%, 425 \mathrm{mV}, 17.23 \mathrm{~mA} / \mathrm{cm}^{2}$, and 0.44 were measured, respectively. Few suggestions were made concerning the paths than can be followed to improve the characteristics of the cell. According to the authors, corrections to light reflection and transmission could be carried out as an effort to achieve better characteristics.

The potential application of SnSe compound in heterostructures has been also demonstrated. Mao et al. studied the electrical properties of a two-dimensional (2D) $\mathrm{GeSe} / \mathrm{SnSe}$ type-II heterojunction as an alternative to single $\mathrm{SnSe}$ and GeSe layers in optoelectronic applications. ${ }^{[12]}$ The resultant junction was reported to have a $0.89 \mathrm{eV}$ band gap and a power conversion efficiency (PCE) of $21.47 \%$ obtained using a computational approach based on Scharber's method. This high value of PCE was attributed to the separation of holes and electrons and their further transit through the junction, process eased by the offset and alignment of the conduction and valence band of the two individual layers acting as a junction, which was determined by the electronic states density in the conduction band minimum (CBM) and valence band maximum (VBM) for each material.

In other work, 2D type-II heterojunctions with good optoelectronic and electrical characteristics have been described. A highly crystalline few-layer Van der Waals $\mathrm{SnSe} / \mathrm{MoS}_{2}$ heterostructure was studied by Yang et al. ${ }^{[13]}$ Such a junction showed excellent electrical transport with a distinct rectification effect as well as a high external quantum efficiency (EQE). Additionally, it showed the capability to generate a self-power photocurrent under illumination due to the separate accumulation of charge carriers in the different constituents of the junction, which limits the electron-hole pairs recombination. Thus, the use of heterostructures stands out as an alternate approach for further improvement to solar cells.

For the sake of better understanding of the reported experimental results, a theoretical analysis is now presented, where the potentiality of $\mathrm{SnSe}$ as absorber material in solar cells is analyzed by the radiative limit, where the only band to band transitions is assumed. It is remarked that under the radiative limit, there are important assumptions such as the ideality of resistances, buffer, and window layers as well as the lack of defects at both absorber material and absorber/buffer interface. In this sense, the maximum expected efficiencies in solar cells consisting of the proposed material as the absorber can be evaluated. Further details on calculations can be found in reference. ${ }^{[14]}$

The impact of SnSe thickness on open-circuit voltage, short-circuit current density, and efficiency is illustrated in Figure 1. It is observed that maximum open-circuit voltage values in the range of $624-702 \mathrm{mV}$ are expected under the radiative limit. Taking the experimental results into account, it is pointed out that $V_{\mathrm{oc}}$ is almost reduced by $300 \mathrm{mV}$, which is a result of the role that the formation of defects is playing, increasing carrier recombination and thereby reducing $V_{\text {oc }}$ of experimental solar cells. An interesting result is that SnSe solar cells can provide maximum $J_{\mathrm{sc}}$ value of $47.6 \mathrm{~mA} / \mathrm{cm}^{2}$ for $\mathrm{SnSe}$ thicknesses higher than $2 \mu \mathrm{m}$. This implies losses of almost 30 $\mathrm{mA} / \mathrm{cm}^{2}$ in experimental cells. In the theoretical calculations, fill factor values in the range of $0.83-0.85$ are found (not shown), while experimental values lower than 0.5 have been reported elsewhere. In addition, maximum efficiency of $25.6 \%$ is expected for a SnSe thickness of $1 \mu \mathrm{m}$ as illustrated in Figure 1. This value has not been reached experimentally due to the reduced $V_{\mathrm{oc}}, J_{\mathrm{sc}}$, and FF reported. In this sense, this value stands for the goal of the scientific community.

From the comparison between results summarized in Ref. [6] for different SnSe solar cells and the theoretically expected values illustrated in Figure 1, it can be concluded that the first limiting factor concerning this technology is the reduced $J_{\text {sc }}$. Some of the factors that produce $J_{\mathrm{sc}}$ losses are reflection, very short minority carrier lifetimes, and diffusion lengths, and a high density of defects that might contribute to the poor carrier collection. In particular, the influence of defects and the presence of non-ideal band-alignment and non-ideal contacts can inhibit carrier transport. If a high defect density is formed, carriers would recombine, thereby reducing carrier lifetime and resulting in poor carrier diffusion length and mobility values. In addition, the presence of an ideal spike-like alignment with a relative low band off-set and ideal contacts are also mandatory to guarantee the correct carrier collection. In this sense, further 


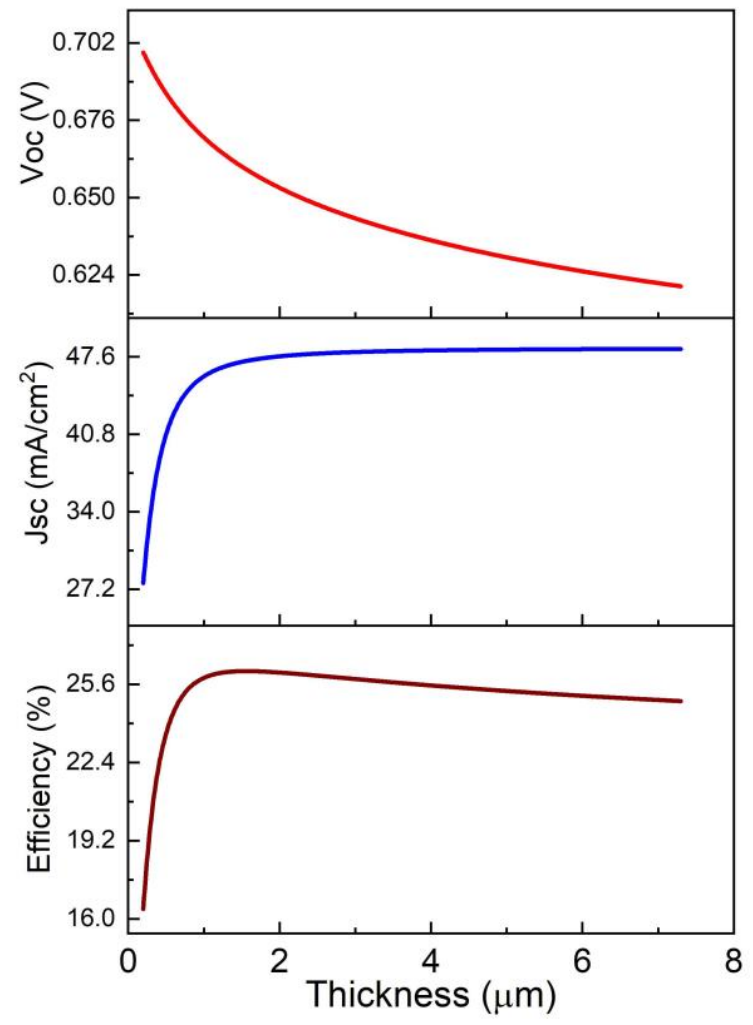

Figure 1 Calculated values of open-circuit voltage $\left(V_{o c}\right)$, short-circuit current density $\left(J_{\mathrm{sc}}\right)$, and efficiency as functions of SnSe thickness.

studies on improving the crystallinity quality of SnSe compound are mandatory. This can result in a reduced density of defects thereby increasing $V_{o c}$ and $J_{s c}$ for the reduction of recombination. Another important aspect is to find an adequate buffer layer with proper spike-like band-alignment with the SnSe material and with lattice constants about the ones of SnSe to avoid losses at the SnSe/buffer interface, which would be translated into a higher $V_{\text {oc }}$ and $J_{\text {sc }}$. Last but not least, fill factor values reported are very low. The value of the fill factor in solar cells is not only determined by $J_{\mathrm{sc}}$ and $V_{\text {oc }}$ but also by series and shunt resistances. Therefore, a minimal contribution from series resistances and the highest shunt resistance are needed. For achieving lower values of series resistances, a detailed study on alternative contacts is required to replace expensive contacts such as $\mathrm{Au}$ and $\mathrm{Ag}$, while further studies are also recommended to improve the $\mathrm{SnSe} /$ buffer interface that would result in improved shunt resistances.

In short, this work presents our perspective on SnSe thin film solar cell technology. It is demonstrated that $\mathrm{SnSe}$ constitutes a potential candidate for the next generation of solar cells since an efficiency of $25.6 \%$ is expected under the ideal conditions. In particular, a low density of defects at SnSe bulk and the SnSe/buffer interface as well as the use of a buffer layer with a high band-gap so that most photons get absorbed in the SnSe material and with good lattice matching with the $\mathrm{SnSe}$ are mandatory. In addition, the negligible contribution of resistances is desirable. All these points constitute a guide for experimentalists working on SnSe solar cells for the further promotion of this technology.

\section{Conflict of Interest}

The authors declare no conflict of interest.

Copyright (c) 2021 Paul Beltrán-Bobadilla, A. Carrillo-Osuna, J. A. Rodriguez-Valverde, B. Acevedo- Juárez, I. Montoya De Los Santos, F. J. Sánchez-Rodriguez, Maykel Courel. This article is an open access article distributed under the terms and conditions of the Creative Commons Attribution (CC BY) license (http://creativecommons.org/ licenses/by/4.0/). The use, distribution or reproduction in other forums is permitted, provided the original author(s) or licensor are credited and that the original publication in this journal is cited, in accordance with accepted academic practice. No use, distribution or reproduction is permitted which does not comply with these terms.

\section{References}

[1] Courel, M.; Arce-Plaza, A.; Oseguera-Galindo, D.; Vigil-Galan, O. The Path to Improve Kesterite Solar Cell Efficiency. Gen. Chem. 2018, 4, 180015

[2] Ju, M.-G.; Chen, M.; Zhou, Y.; Dai, J.; Ma, L.; Padture, N. P.; Zeng, $X$. C. Toward Eco-friendly and Stable Perovskite Materials for Photovoltaics. Joule 2018, 2, 1231-1241.

[3] Shi, W.; Gao, M.; Wei, J.; Gao, J.; Fan, C.; Ashalley, E.; Wang, Z. Tin Selenide (SnSe): Growth, Properties, and Applications. Adv. Sci. 2018, 5, 1700602.

[4] Im, H. S.; Myung, Y.; Park, K.; Jung, C. S.; Lim, Y. R.; Jang, D. M.; Park, J. Ternary alloy nanocrystals of tin and germanium chalcogenides. RSC Adv. 2014, 4, 15695-15701.

[5] Huang, Y.; Wang, C.; Chen, X.; Zhou, D.; Du, J.; Wang, S.; Ning, L. First-principles study on intrinsic defects of SnSe. RSC Adv. 2017, 7, 27612-27618

[6] Reddy, V. M. R.; Gedi, S.; Pejjai, B.; Park, C. Perspectives on SnSe-based thin film solar cells: a comprehensive review. J. Mater. Sci. Mater. Electron. 2016, 27, 5491-5508.

[7] Singh, J. P.; Bedi, R. K. FTO/SnSe Heterojunction for Photovoltaic Conversion. Jpn. J. Appl. Phys. 1990, 29, L792.

[8] Mathews, N. R. Electrodeposited tin selenide thin films for photovoltaic applications. Sol. Energy 2012, 86, 1010-1016.

[9] Shinde, D. V.; Min, S. K.; Sung, M. M.; Shrestha, N. K.; Mane, R. S.; Han, S. H. Photovoltaic properties of nanocrystalline SnSe-CdS. Mater. Lett. 2014, 115, 244-247.

[10] Makori, N. E.; Amatalo, I. A.; Karimi, P. M.; Njoroge, W. K. Characterization of SnSe-CdO:Sn P-N Junction for Solar Cell Applications. Int. J. Energy Eng. 2015, 5, 1-4.

[11] Abd El-Rahman, K. F.; Darwish A. A. A.; El-Shazly, E. A. A. Electrical and photovoltaic properties of $\mathrm{SnSe} / \mathrm{Si}$ heterojunction. Mater. Sci. Semicond. Process. 2014, 25, 123-129.

[12] Mao, Y.; Xu, C.; Yuan, J.; Zhao, H. A two-dimensional GeSe/SnSe heterostructure for high performance thin-film solar cells. J. Mater. Chem. A 2019, 7, 11265-11271.

[13] Yang, S.; Wu, M.; Wang, B.; Zhao, L.-D.; Huang, L.; Jiang, C.; Wei, S.-H. Enhanced Electrical and Optoelectronic Characteristics of Few-Layer Type-II SnSe/MoS 2 van der Waals Heterojunctions. ACS Appl. Mater. Interfaces 2017, 9, 42149-42155.

[14] Jiménez, T.; Seuret-Jiménez, D.; Vigil-Galán, O.; Basurto-Pensado, M. A.; Courel, M. $\mathrm{Sb}_{2}\left(\mathrm{~S}_{1-x} \mathrm{Se}_{x}\right)_{3}$ solar cells: the impact of radiative and non-radiative loss mechanisms. J. Phys. D Appl. Phys. 2018, $51,435501$. 